\title{
Prolongement dans des classes ultradifférentiables et propriétés de régularité des compacts de $\mathbb{R}^{n}$
}

\author{
par VinCEnt ThiLliez (Lille)
}

\begin{abstract}
Considering jets, or functions, belonging to some strongly non-quasianalytic Carleman class on compact subsets of $\mathbb{R}^{n}$, we extend them to the whole space with a loss of Carleman regularity. This loss is related to geometric conditions refining Łojasiewicz's "regular separation" or Whitney's "property (P)".
\end{abstract}

0. Introduction. Dans un premier temps, on étudie ici l'extension simultanée (ou, en d'autres termes, le recollement) de deux jets de Whitney $F_{1}$ et $F_{2}$ appartenant à une classe de Carleman fortement non-quasianalytique sur des compacts respectifs $E_{1}, E_{2}$ de $\mathbb{R}^{n}$, et coïncidant sur $E_{1} \cap E_{2}$. On démontre en 2.4 l'équivalence d'une propriété d'extension avec perte de régularité (passage de $l ! M_{l}$ à $l ! N_{l}$ ) et d'une certaine inégalité de Łojasiewicz raffinée pour $E_{1}, E_{2}$. En particulier, lorsque $E_{1}, E_{2}$ sont régulièrement situés au sens usuel $([\mathrm{M}],[\mathrm{T}])$, on a $N_{l}=M_{l}^{m}$ où $m$ est l'exposant de l'inégalité de Łojasiewicz. (Dans le cas classique de l'extension de jets $C^{\infty}$, la perte de régularité est occultée : voir $[\mathrm{T}]$, chapitre $\mathrm{IV}$, ou $[\mathrm{M}]$ ).

A titre d'exemple, si on a, pour tout $x$ d'un voisinage de $E_{1} \cup E_{2}$, l'inégalité

$$
d\left(x, E_{1}\right)+d\left(x, E_{2}\right) \geq \gamma \frac{d\left(x, E_{1} \cap E_{2}\right)^{m}}{\left|\log d\left(x, E_{1} \cap E_{2}\right)\right|^{p}}
$$

avec $\gamma>0, m \geq 1, p \geq 0$, et si $F_{1}$ et $F_{2}$ ont la régularité Gevrey $l^{1+\alpha}(\alpha>0)$, alors il existe une fonction ayant la régularité Carleman $l !^{1+m \alpha}(\log l)^{l p}$ sur $\mathbb{R}^{n}$ et dont le jet sur $E_{j}$ coïncide avec $F_{j}(j=1,2)$. Réciproquement, un tel phénomène d'extension impose l'inégalité $(*)$.

Les propriétés ainsi décrites permettent notamment de préciser un exemple de W. Pleśniak [P] concernant le prolongement de fonctions ultra-

1991 Mathematics Subject Classification: 26E10, 58C25.

Key words and phrases: Carleman class, extension theorem, Łojasiewicz inequalities, Whitney regularity. 
différentiables lorsqu'on omet la condition de reste de Taylor sur les jets associés.

Dans un deuxième temps, on revient à ce dernier problème par le biais d'une propriété géométrique raffinant la propriété $(\mathrm{P})$ de Whitney. On obtient ainsi en 3.2 un théorème d'extension de fonctions, sans considération de jets de Whitney, qui étend, en un certain sens, un résultat de J. Bonet, R. W. Braun, R. Meise et B. A. Taylor ([BBMT], 3.12). Par exemple, si $\Omega$ est un ouvert borné d'adhérence $m$-régulière $(m \geq 1)$ au sens usuel de Whitney ([M], $[\mathrm{T}])$, toute fonction ayant la régularité Carleman $l ! M_{l}$ sur $\Omega$ s'étend en une fonction ayant la régularité $l ! M_{l}^{m}$ sur $\mathbb{R}^{n}$.

Il est à noter que dans $[\mathrm{P}]$, la question du prolongement de fonctions ultradifférentiables est abordée sous un angle a priori différent (classes de fonctions définies par approximation polynômiale).

L'auteur tient enfin à remercier J. Chaumat et A.-M. Chollet pour une discussion utile au sujet de la proposition 2.2.

\section{Classes de Carleman}

1.1. Suites fortement régulières. On dira qu'une suite $M=\left(M_{l}\right)_{l>0}$ de réels positifs est fortement régulière lorsqu'elle satisfait les propriétés suivantes :

$$
M_{0}=1, M \text { est croissante, }\left(M_{l+1} / M_{l}\right)_{l \geq 0} \text { est croissante, }
$$

et il existe une constante $A \geq 1$ telle que l'on ait, pour tout $l$,

$$
M_{l} \leq A^{l} M_{j} M_{l-j} \quad \text { pour } 0 \leq j \leq l
$$

et

$$
\sum_{j \geq l} \frac{M_{j}}{(j+1) M_{j+1}} \leq A l \frac{M_{l}}{(l+1) M_{l+1}} .
$$

Lorsque ces conditions sont vérifiées, il est prouvé dans $[\mathrm{CC}]$ que l'on a $M_{j} M_{k} \leq M_{j+k}$ pour tous entiers $j$ et $k$, que $\left(M_{l+1} / M_{l}\right)_{l \geq 0}$ et $\left(M_{l}^{1 / l}\right)_{l \geq 0}$ croissent vers $\infty$ et que l'on a, quitte à augmenter $A$,

$$
M_{l+1}^{l} \leq A^{l} M_{l}^{l+1}
$$

pour tout entier $l$.

Posons par ailleurs

$$
h_{M}(t):=\inf _{j \geq 0} t^{j} M_{j} \quad \text { pour } t \in \mathbb{R}_{+} .
$$

La fonction $h_{M}$ est continue, croissante, on a $h_{M}(0)=0$ et $h_{M}(t)=1$ pour $t \geq 1$. En outre, d'après [CC], il existe une constante $B \geq 1$ telle que l'on ait

$$
h_{M}(t / B) \leq\left(h_{M}(t)\right)^{2}
$$


Si $m$ est un réel fixé, $m \geq 1$, il est facile de voir que la suite $M^{m}:=$ $\left(M_{l}^{m}\right)_{l \geq 0}$ est aussi fortement régulière et que l'on a

$$
\left(h_{M}(t)\right)^{m}=h_{M^{m}}\left(t^{m}\right) .
$$

Pour tout entier $l$ et tout $t$ de $\mathbb{R}_{+}$, on pose encore

$$
h_{M}(l, t):=\inf _{j \geq l} t^{j} M_{j} .
$$

En particulier, on a $h_{M}(0, t)=h_{M}(t)$. Les fonctions $h_{M}(l, \cdot)$ seront utilisées au $\S 3$.

Notations. Pour tout multi-indice $L=\left(l_{1}, \ldots, l_{n}\right)$ de $\mathbb{N}^{n}$, on notera $l=|L|=l_{1}+\ldots+l_{n}$ la longueur de $L$ et $D^{L}=\partial^{l} / \partial x_{1}^{l_{1}} \ldots \partial x_{n}^{l_{n}}$ le monôme de dérivation associé à $L$. On posera aussi $L$ ! $=l_{1} ! \ldots l_{n}$ ! et, pour tout $x=\left(x_{1}, \ldots, x_{n}\right)$ de $\mathbb{R}^{n}, x^{L}=x_{1}^{l_{1}} \ldots x_{n}^{l_{n}}$.

1.2. Fonctions de classe $C_{M}$. Soient $\Omega$ un ouvert quelconque de $\mathbb{R}^{n}$ et $M$ une suite fortement régulière.

Ici, $C^{\infty}(\bar{\Omega})$ désignera l'espace des fonctions indéfiniment différentiables à l'intérieur de $\bar{\Omega}$ et dont toutes les dérivées se prolongent continûment à $\bar{\Omega}$ (et non l'espace $J^{\infty}(\bar{\Omega})$ des fonctions $C^{\infty}$ au sens de Whitney, ou "jets de Whitney $C^{\infty}$ ", sur $\bar{\Omega}$, bien que le choix entre les notations varie suivant les auteurs).

Une fonction $f$ de $C^{\infty}(\bar{\Omega})$ sera dite appartenir à la classe de Carleman $C_{M}(\bar{\Omega})$ s'il existe une constante positive $C$ (dépendant de $f$ ) telle que l'on ait, pour tout multi-indice $L$ et tout $x$ de $\Omega$,

$$
\left|D^{L} f(x)\right| \leq C^{l+1} l ! M_{l} .
$$

La classe $C_{M}(\bar{\Omega})$ est une algèbre; (1.1.2) en traduit la stabilité par opérateurs (ultra)différentiels et (1.1.3) la forte non-quasianalyticité [B].

1.3. Classes de jets. Soient $E$ un compact de $\mathbb{R}^{n}$ et $M$ une suite fortement régulière.

Un jet sur $E$ est la donnée d'une famille $F=\left(F_{L}\right)_{L \in \mathbb{N}^{n}}$ de fonctions continues sur $E$. Au jet $F$ on associe, pour tout entier $p$ et tout $(\xi, x)$ de $E \times \mathbb{R}^{n}$, le polynôme de Taylor

$$
T_{\xi}^{p} F(x):=\sum_{J, 0 \leq j \leq p} \frac{1}{J !} F_{J}(\xi)(x-\xi)^{J} .
$$

On dit alors que $F$ appartient à la classe de Carleman de jets $J_{M}(E)$ s'il existe une constante positive $C$ telle que l'on ait, pour tout multi-indice $J$ et tout $\xi$ de $E$,

$$
\left|F_{J}(\xi)\right| \leq C^{j+1} j ! M_{j}
$$

et, pour tout entier $p$, tout multi-indice $L$ avec $l \leq p$ et tout $x$ de $E$,

$$
\left|F_{L}(x)-D^{L} T_{\xi}^{p} F(x)\right| \leq C^{p+1} l ! M_{p+1}|x-\xi|^{p+1-l} .
$$


Si $\Omega$ est un ouvert contenant $E$, toute fonction de $C_{M}(\bar{\Omega})$ définit un jet de $J_{M}(E)$ via l'application de Borel

$$
\Re_{E}: C_{M}(\bar{\Omega}) \rightarrow J_{M}(E), \quad f \rightarrow\left(\left.D^{L} f\right|_{E}\right)_{L \in \mathbb{N}^{n}} .
$$

Si $\widetilde{E}$ est un compact contenant $E$ et si $F$ est un jet de $J_{M}(\widetilde{E})$, on notera encore $\Re_{E} F$ la restriction de $F$ à $E$, c'est-à-dire le jet $\left(\left.F_{L}\right|_{E}\right)_{L \in \mathbb{N}^{n}}$. On utilisera le résultat suivant, qui traduit la surjectivité de l'application (1.3.1) :

ThÉORÈme 1.4 ([B], [CC]). Avec les notations précédentes, soit $F$ un jet de $J_{M}(E)$. Il existe une fonction $f$ de $C_{M}\left(\mathbb{R}^{n}\right)$, à support compact, telle que l'on ait $\Re_{E} f=F$.

1.5. Re mar que ([BBMT], 3.12). On rappelle qu'un compact $E$ de $\mathbb{R}^{n}$ a la propriété $(\mathrm{P})$ de Whitney s'il existe une constante positive $\gamma$ telle que deux points quelconques $\xi$ et $x$ de $E$ puissent être joints par un arc rectifiable $\sigma$ contenu dans l'intérieur de $E$ sauf peut-être pour un nombre fini de points, et dont la longueur $|\sigma|$ vérifie l'inégalité $|\sigma| \leq \gamma|x-\xi|$.

Soit $\Omega$ un ouvert borné tel que $\bar{\Omega}$ ait la propriété $(\mathrm{P})$ de Whitney. Alors toute fonction de $C_{M}(\bar{\Omega})$ définit un jet de $J_{M}(\bar{\Omega})$ et s'étend donc en une fonction de $C_{M}\left(\mathbb{R}^{n}\right)$, en vertu de (1.4).

1.6. DÉfinition. Une fonction $\theta: \mathbb{R}_{+} \rightarrow \mathbb{R}_{+}$sera dite admissible lorsqu'elle satisfait les propriétés suivantes :

$$
\theta \text { est croissante et } \theta(0)=0,
$$

pour tout réel $\lambda$ avec $\lambda \geq 1$, la quantité

$$
\bar{\theta}(\lambda):=\sup _{0<t \leq 1} \theta(\lambda t) / \theta(t)
$$

est finie, et il existe une constante $a \geq 1$ telle que l'on ait

$$
\theta(t) \leq a t \quad \text { pour } 0 \leq t \leq 1
$$

On remarquera comme conséquence immédiate de (1.6.1)-(1.6.3) que pour $T \geq 1$ et $0 \leq t \leq T$, on a

$$
\begin{gathered}
\theta(s+t) \leq \bar{\theta}(2)(\theta(s)+\theta(t)) \quad \text { pour } 0 \leq t \leq 1,0 \leq s \leq 1, \\
\theta(\lambda t) \leq \bar{\theta}(T \lambda) \theta(t)
\end{gathered}
$$

et

$$
\theta(t) \leq a \frac{\bar{\theta}(T)}{T} t
$$

Remarque. Soient une fonction admissible $\theta$ et un réel $\lambda>1$. En considérant une partition de $] 0,1]$ en intervalles $\left.\left.I_{j}:=\right] \lambda^{-(j+1)}, \lambda^{-j}\right]$ et en utilisant de façon répétée (1.6.2), on obtient, pour $t \in I_{j}$, l'inégalité $\theta(t) \geq$ $\theta\left(\lambda^{j} t\right) \bar{\theta}(\lambda)^{-j}$ avec $\lambda^{j} t \geq 1 / \lambda$ et $j \leq(\log t) / \log \lambda$. Il s'ensuit que l'on a, pour 
tout $t$ de $[0,1]$,

$$
\theta(t) \geq \theta(1 / \lambda) t^{m(\lambda)}
$$

avec $m(\lambda)=(\log \bar{\theta}(\lambda)) / \log \lambda$. Compte tenu de (1.6.3), on a par ailleurs $m(\lambda) \geq 1$.

\section{Extension simultanée}

2.1. Définitions. Soient $E_{1}$ et $E_{2}$ deux compacts de $\mathbb{R}^{n}$ et soit $\theta$ une fonction admissible. Si on a $E_{1} \cap E_{2} \neq \emptyset$, on dit que $E_{1}$ et $E_{2}$ sont $\theta$-situés lorsqu'il existe un ouvert borné $\Omega$ contenant $E_{1} \cup E_{2}$ et une constante $\gamma>0$, ne dépendant que de $E_{1}, E_{2}$, tels que l'on ait, pour tout $x$ de $\Omega$,

$$
d\left(x, E_{1}\right)+d\left(x, E_{2}\right) \geq \gamma \theta\left(d\left(x, E_{1} \cap E_{2}\right)\right) .
$$

Si on a $E_{1} \cap E_{2}=\emptyset$, on dit encore que $E_{1}$ et $E_{2}$ sont $\theta$-situés, avec $\theta(t)=t$.

Par exemple, soit $m$ un réel, $m \geq 1$. La fonction $\theta(t)=t^{m}$ est admissible et on voit que $E_{1}$ et $E_{2}$ sont $\theta$-situés si et seulement si ils sont régulièrement situés $([\mathrm{M}],[\mathrm{T}])$ avec un exposant de Łojasiewicz égal à $m$.

En fait, il résulte de (1.6.7) que deux compacts $\theta$-situés sont toujours régulièrement situés : (2.1.1) peut donc être vu comme une inégalité de Łojasiewicz raffinée, l'échelle de précision n'étant pas limitée aux fonctions $t \rightarrow t^{m}$ (voir l'exemple (ii) ci-après).

Soient alors $\theta$ une fonction admissible et $M, N$ deux suites fortement régulières. On dira que la condition $(\mathcal{C})$ est vérifiée s'il existe des constantes $b, c$, avec $0<b<1,0<c<1$, telles que l'on ait

$$
b h_{M}(c t) \leq h_{N}(\theta(t)) \leq b^{-1} h_{M}\left(c^{-1} t\right)
$$

pour tout $t$ de $\mathbb{R}_{+}$.

R e marque. Pour toute suite fortement régulière $M$ (ou, plus généralement, vérifiant (1.1.1)), on a, pour tout entier $l$,

$$
C^{-(l+1)} M_{l} \leq \sup _{0<t \leq 1} h_{M}(t) / t^{l} \leq C^{l+1} M_{l}
$$

pour une constante $C$ convenable.

Comme, d'après (1.6.6) et (2.1.2), on a $h_{M}(t) \leq b^{-1} h_{N}\left(\bar{\theta}\left(c^{-1}\right) a t\right)$ pour $0 \leq t \leq 1$, de la condition $(\mathcal{C})$ va résulter l'inégalité

$$
\sup _{l \geq 0}\left(M_{l} / N_{l}\right)^{1 / l}<\infty
$$

Exemples. (i) Soient $m$ un réel, $m \geq 1$, et $\theta(t)=t^{m}$. Il résulte facilement de (1.1.7) et d'une application répétée de (1.1.6) que si $M$ est une suite fortement régulière quelconque, alors $(\mathcal{C})$ est vérifiée avec $N=M^{m}$.

(ii) Comme généralisation de l'exemple (i), on peut considérer la fonction admissible $\theta(t)=t^{m}(\log (1+1 / t))^{-p}$ avec $m$ et $p$ réels, $m \geq 1, p \geq 0$. 
Alors, si $M$ est la suite de Gevrey $M_{l}=l !^{\alpha}, \alpha>0$, et si $N$ est définie par $N_{l}=l !^{m \alpha}(\log l)^{l p}$, des calculs élémentaires permettent de montrer que $(\mathcal{C})$ est vérifiée.

Dans l'exemple (ii), ou plus généralement avec toute fonction admissible $\theta$, il est naturel de se demander si, étant donnée une suite $M$ fortement régulière quelconque, il existe toujours une suite $N$ fortement régulière telle que $(\mathcal{C})$ soit vérifiée. La proposition suivante répond par l'affirmative, sous des hypothèses supplémentaires de régularité pour $\theta$, peu restrictives. La preuve fournit en outre une construction explicite de $N$.

2.2. Proposition. Soient $M$ une suite fortement régulière et $\theta$ une fonction admissible satisfaisant les propriétés suivantes au voisinage de 0 dans $\mathbb{R}_{+}$:

$$
\begin{aligned}
& \text { la fonction } t \rightarrow \theta(t) / t \text { est croissante, } \\
& i l \text { existe un entier } q>1 \text { tel que } t \rightarrow \theta(t) / t^{q} \text { soit décroissante. }
\end{aligned}
$$

Alors il existe une suite $N$ fortement régulière telle que la condition $(\mathcal{C})$ soit vérifiée.

Preuve. On pose $m_{l}:=M_{l+1} / M_{l}$ de sorte que l'on a $M_{l}=m_{0} \ldots m_{l-1}$ pour tout $l \geq 1$. On définit alors $N$ par les égalités $N_{0}=1$ et $N_{l}=n_{0} \ldots n_{l-1}$ pour $l \geq 1$, avec

$$
n_{l}:=\frac{1}{\theta\left(1 / m_{l}\right)} .
$$

On vérifie d'abord que $N$ est fortement régulière. D'après (1.1), on sait que $\left(m_{l}\right)_{l \geq 0}$ croît vers $\infty$. On voit immédiatement qu'il en est de même pour $\left(n_{l}\right)_{l \geq 0}$, donc que $N$ satisfait (1.1.1). Écrivons ensuite $n_{l}=\varepsilon_{l} m_{l}^{q}$, où $q$ est l'entier qui figure dans $(2.2 .2)$ et où la suite $\left(\varepsilon_{l}\right)_{l \geq 0}$, donnée par

$$
\varepsilon_{l}:=\frac{\left(1 / m_{l}\right)^{q}}{\theta\left(1 / m_{l}\right)}
$$

est en conséquence décroissante (on peut supposer sans perte de généralité que $(2.2 .2)$ a lieu sur $\left.\left.] 0,1 / m_{0}\right]\right)$. On a alors, pour tout entier $l$,

$$
N_{l}=\varepsilon_{0} \ldots \varepsilon_{l-1} M_{l}^{q}
$$

et, puisque $M$ satisfait (1.1.2),

$$
N_{l} \leq A^{q l} \varepsilon_{0} \ldots \varepsilon_{l-1} M_{j}^{q} M_{l-j}^{q}
$$

pour $0 \leq j \leq l$. On a en outre $\varepsilon_{0} \ldots \varepsilon_{j-1} \varepsilon_{j} \ldots \varepsilon_{l-1} \leq \varepsilon_{0} \ldots \varepsilon_{j-1} \varepsilon_{0} \ldots \varepsilon_{l-j-1}$ puisque $\left(\varepsilon_{l}\right)_{l \geq 0}$ est décroissante. En reportant dans (2.2.5), on obtient

$$
N_{l} \leq\left(A^{q}\right)^{l}\left(\varepsilon_{0} \ldots \varepsilon_{j-1} M_{j}^{q}\right)\left(\varepsilon_{0} \ldots \varepsilon_{l-j-1} M_{l-j}^{q}\right),
$$


donc, compte tenu de (2.2.4), que $N$ satisfait (1.1.2). Enfin, on a

$$
\begin{aligned}
\sum_{j \geq l} \frac{N_{j}}{(j+1) N_{j+1}} & =\sum_{j \geq l} \frac{1}{(j+1) n_{j}}=\sum_{j \geq l} \frac{\theta\left(1 / m_{j}\right)}{j+1} \\
& =\sum_{j \geq l} \frac{1}{(j+1) m_{j}} \cdot \frac{\theta\left(1 / m_{j}\right)}{1 / m_{j}} \leq \frac{\theta\left(1 / m_{l}\right)}{1 / m_{l}} \sum_{j \geq l} \frac{1}{(j+1) m_{j}},
\end{aligned}
$$

d'après $\left(2.2 .1\right.$ ) et la décroissance de $\left(1 / m_{l}\right)_{l \geq 0}$ (on peut également supposer que $(2.2 .1)$ a lieu sur $\left.\left.] 0,1 / m_{0}\right]\right)$. L'hypothèse $(1.1 .3)$ pour $M$ se traduit par

Il vient donc

$$
\sum_{j \geq l} \frac{1}{(j+1) m_{j}} \leq A l \frac{1}{(l+1) m_{l}} .
$$

$$
\sum_{j \geq l} \frac{N_{j}}{(j+1) N_{j+1}} \leq A l \frac{\theta\left(1 / m_{l}\right)}{l+1}=A l \frac{N_{l}}{(l+1) N_{l+1}},
$$

c'est-à-dire que $N$ satisfait (1.1.3).

On établit maintenant la condition $(\mathcal{C})$. On découpe $\left.I:=] 0,1 / m_{0}\right]$ en intervalles $\left.\left.I_{l}:=\right] 1 / m_{l}, 1 / m_{l-1}\right], l \geq 1$. Il est élémentaire de vérifier que l'on a

$$
h_{M}(t)=t^{l} M_{l} \quad \text { pour } t \in I_{l}
$$

et $\theta(t) \in\left[1 / n_{l}, 1 / n_{l-1}\right]$, donc, pour la même raison,

$$
h_{N}(\theta(t))=\theta(t)^{l} N_{l} \quad \text { pour } t \in I_{l} .
$$

Enfin, d'après (2.2.1), on a

$$
\left(\frac{\theta(t)}{t}\right)^{l} N_{l} \geq\left(\frac{\theta\left(1 / m_{l}\right)}{1 / m_{l}}\right)^{l} N_{l} \geq\left(\frac{m_{l}}{n_{l}}\right)^{l} N_{l} \quad \text { pour } t \in I_{l} .
$$

Par ailleurs, comme $N$ est fortement régulière, elle satisfait la propriété (1.1.4). En d'autres termes, on a $n_{l}^{l} \leq C^{l} N_{l}$ pour une constante $C$ convenable, $C \geq 1$. L'inégalité $M_{l}=m_{0} \ldots m_{l-1} \leq m_{l}^{l}$ étant triviale puisque $\left(m_{l}\right)_{l \geq 0}$ croît, on obtient $\left(m_{l} / n_{l}\right)^{l} \geq C^{-l} M_{l} / N_{l}$ et, par (2.2.7) et (2.2.8), $h_{N}(\theta(t)) \geq(t / C)^{l} M_{l}$ pour $t \in I_{l}$. Comme on a, par définition, $h_{M}(t / C) \leq$ $(t / C)^{l} M_{l}$ pour tout $t$, il s'ensuit

$$
h_{M}(t / C) \leq h_{N}(\theta(t)) \quad \text { pour tout } t \text { de } I .
$$

En vertu de (2.2.1), on a aussi

$$
\left(\frac{\theta(t)}{t}\right)^{l} N_{l} \leq\left(\frac{\theta\left(1 / m_{l-1}\right)}{1 / m_{l-1}}\right)^{l} N_{l}=\left(\frac{m_{l-1}}{n_{l-1}}\right)^{l} N_{l} \quad \text { pour } t \in I_{l} .
$$

En utilisant, similairement à ce qui précède, (1.1.4) et la croissance de $\left(n_{l}\right)_{l \geq 0}$, on obtient $\left(m_{l-1} / n_{l-1}\right)^{l} \leq\left(C^{\prime}\right)^{l} M_{l} / N_{l}$ et donc, par (2.2.6) et 
(2.2.10), $\left(\theta(t) / C^{\prime}\right)^{l} N_{l} \leq h_{M}(t)$ pour $t \in I_{l}, C^{\prime}$ désignant une constante convenable, avec $C^{\prime} \geq 1$. Il s'ensuit

$$
h_{N}\left(\theta(t) / C^{\prime}\right) \leq h_{M}(t) \quad \text { pour tout } t \text { de } I .
$$

Enfin, d'après $(2.2 .1)$, on a $\theta\left(C^{\prime} t\right) / C^{\prime} \geq \theta(t)$ et, en reportant dans (2.2.11), $h_{N}(\theta(t)) \leq h_{N}\left(\theta\left(C^{\prime} t\right) / C^{\prime}\right) \leq h_{M}\left(C^{\prime} t\right)$ pour $t \in\left(1 / C^{\prime}\right) I$. Joint à $(2.2 .9)$, ceci montre que l'on a $(2.1 .2)$ sur un intervalle $\left[0, t_{0}\right]\left(t_{0}>0\right)$, ce qui suffit à prouver $(\mathcal{C})$.

2.3. Lemme. Soient $E_{1}$ et $E_{2}$ deux compacts de $\mathbb{R}^{n}$ avec $E_{1} \cap E_{2} \neq \emptyset$, $\theta$ une fonction admissible et $M, N$ deux suites fortement régulières. On suppose que $E_{1}$ et $E_{2}$ sont $\theta$-situés et que la condition $(\mathcal{C})$ est satisfaite. Alors il existe deux ouverts $\Omega_{1}$ et $\Omega_{2}$ contenus dans $\mathbb{R}^{n} \backslash\left(E_{1} \cap E_{2}\right)$ et, pour tout $\lambda>0$, une fonction $\psi_{\lambda}$ de classe $C^{\infty}$ sur $\mathbb{R}^{n} \backslash\left(E_{1} \cap E_{2}\right)$, tels que les propriétés suivantes soient vérifiées :

$$
\begin{gathered}
0 \leq \psi_{\lambda} \leq 1 \quad \text { sur } \mathbb{R}^{n} \backslash\left(E_{1} \cap E_{2}\right), \\
E_{1} \backslash E_{2} \subset \Omega_{1} \quad \text { et } \quad \psi_{\lambda} \equiv 1 \quad \text { sur } \Omega_{1}, \\
E_{2} \backslash E_{1} \subset \Omega_{2} \quad \text { et } \quad \psi_{\lambda} \equiv 0 \quad \text { sur } \Omega_{2},
\end{gathered}
$$

pour tout multi-indice $L$ et tout $x$ de $\mathbb{R}^{n} \backslash\left(E_{1} \cap E_{2}\right)$, on a

$$
\left|D^{L} \psi_{\lambda}(x)\right| \leq C(\lambda)^{l+1} l ! N_{l}\left(h_{M}\left(\lambda d\left(x, E_{1} \cap E_{2}\right)\right)\right)^{-1},
$$

où $C(\lambda)$ est une constante positive ne dépendant que de $\lambda$, de la géométrie de $E_{1}, E_{2}$ (en particulier de $\theta$ ) et des suites $M, N$.

Preuve. On commence par construire deux familles homothétiques de boules euclidiennes $Q_{j}=B\left(x^{j}, r_{j}\right), Q_{j}^{*}=B\left(x^{j},(1+\delta) r_{j}\right), j \in \mathbb{N}$ (où $\delta$ est une constante positive convenable) avec $Q_{j} \cap E_{1} \neq \emptyset$, satisfaisant les propriétés suivantes :

$$
\text { on a } E_{1} \backslash E_{2} \subset \bigcup_{j \geq 0} Q_{j} \text { et } E_{2} \cap \overline{\bigcup_{j \geq 0} Q_{j}^{*}} \subset E_{1} \cap E_{2} \text {, }
$$

il existe un entier $\mathcal{N}$ tel que pour tout $i$ de $\mathbb{N}$,

la boule $Q_{i}^{*}$ coupe au plus $\mathcal{N}$ boules $Q_{j}^{*}, j \neq i$, pour tous $j \in \mathbb{N}$ et $x \in Q_{j}^{*}$, on a

$$
\delta^{\prime} r_{j} \leq d\left(x, E_{2}\right) \leq \delta^{\prime \prime} r_{j} \text { et } d\left(x, E_{1}\right) \leq \delta^{\prime \prime} r_{j},
$$

où $\delta^{\prime}, \delta^{\prime \prime}$ sont des constantes positives convenables.

Pour cela, on obtient les $Q_{j}, Q_{j}^{*}$ en ne conservant, dans un recouvrement de Whitney de $\mathbb{R}^{n} \backslash E_{2}$ ([CC], prop. 5), que les boules qui rencontrent $E_{1}$.

Dans 2.1, on peut clairement supposer, quitte à diminuer $\gamma$, que $\Omega$ contient $\overline{\bigcup_{j>0} Q_{j}^{*}}$.

Soit $\bar{\lambda}>0$, quelconque. En appliquant à $Q_{j}, Q_{j}^{*}$ et à la suite $N$ une construction de J. Bruna ([B], $\S 2$, voir aussi $[\mathrm{CC}]$, prop. 4), on produit alors 
une fonction $\varphi_{\lambda, j}$ de classe $C^{\infty}$ dans $\mathbb{R}^{n}$ satisfaisant :

$$
0 \leq \varphi_{\lambda, j} \leq 1, \operatorname{supp} \varphi_{\lambda, j} \subset Q_{j}^{*} \text { et } \varphi_{\lambda, j} \equiv 1 \text { sur } Q_{j},
$$

pour tout multi-indice $L$ et tout $x$ de $\mathbb{R}^{n}$, on a

$$
\left|D^{L} \varphi_{\lambda, j}(x)\right| \leq\left(C^{\prime} \bar{\theta}(T \lambda)\right)^{l+1} l ! N_{l}\left(h_{N}\left(C \bar{\theta}(T \lambda) r_{j}\right)\right)^{-1},
$$

où on a posé $C=2 \delta^{\prime \prime} / \gamma(\gamma$ est la constante figurant en (2.1.1)), où $T$ est un paramètre, $T \geq 1$, à déterminer, et où $C^{\prime}$ est une constante convenable, ne dépendant que de $N$ et de la géométrie de $E_{1}, E_{2}$.

Observons maintenant que d'après $(1.6 .5),(2.1 .1)$ et $(2.3 .7)$, on a, pour $x \in \operatorname{supp} \varphi_{\lambda, j}$,

$$
h_{N}\left(C \bar{\theta}(T \lambda) r_{j}\right) \geq h_{N}\left(\theta\left(\lambda d\left(x, E_{1} \cap E_{2}\right)\right)\right),
$$

pour une valeur convenable du paramètre $T$. Compte tenu de (2.1.2), on obtient

$$
h_{N}\left(C \bar{\theta}(T \lambda) r_{j}\right) \geq b h_{M}\left(c \lambda d\left(x, E_{1} \cap E_{2}\right)\right),
$$

pour tout $x$ de $\operatorname{supp} \varphi_{\lambda, j}$.

On pose alors $\psi_{\lambda, 1}=\varphi_{\lambda, 1}, \psi_{\lambda, j}=\varphi_{\lambda, j} \prod_{i=1}^{j-1}\left(1-\varphi_{\lambda, i}\right)$ pour $j>1$, et enfin $\psi_{\lambda}=\sum_{j=1}^{\infty} \psi_{\lambda, j}$. Des arguments similaires à ceux de [CC], prop. 6, joints aux propriétés $(2.3 .5),(2.3 .6),(2.3 .8)$ à (2.3.10), permettent de vérifier que $\psi_{\lambda}$ satisfait les conclusions du lemme (quitte à remplacer $\lambda$ par $\lambda / c$ ), avec $\Omega_{1}=\bigcup_{j \geq 0} Q_{j}$ et $\Omega_{2}=\Omega \backslash \overline{\bigcup_{j \geq 0} Q_{j}^{*}}$.

On a alors un théorème concernant l'extension simultanée de deux jets de classe $J_{M}$ sur $E_{1}$ et $E_{2}$.

2.4. ThÉORÈmE. Soient $E_{1}, E_{2}$ deux compacts de $\mathbb{R}^{n}, \theta$ une fonction admissible et $M, N$ deux suites fortement régulières telles que la condition $(\mathcal{C})$ soit vérifiée. Alors les propriétés $(\mathrm{A})$ et $(\mathrm{B})$ suivantes sont équivalentes:

(A) Les compacts $E_{1}$ et $E_{2}$ sont $\theta$-situés.

(B) Quels que soient les jets de Whitney $F_{1}, F_{2}$ satisfaisant

(i) $F_{j} \in J_{M}\left(E_{j}\right), j=1,2$, et

(ii) $\Re_{E_{1} \cap E_{2}} F_{1}=\Re_{E_{1} \cap E_{2}} F_{2}$,

il existe une fonction $f$ de $C_{N}\left(\mathbb{R}^{n}\right)$ telle que l'on ait $\Re_{E_{j}} f=F_{j}$ pour $j=1,2$.

Preuve. $(\mathrm{A}) \Rightarrow(\mathrm{B})$. D'après 1.4, il existe deux fonctions $f_{1}, f_{2}$ de $C_{M}\left(\mathbb{R}^{n}\right)$, vérifiant $\Re_{E_{j}} f_{j}=F_{j}$ pour $j=1,2$. Dans le cas particulier $E_{1} \cap$ $E_{2}=\emptyset$ de la définition 2.1, on prend simplement $f=\psi f_{1}+(1-\psi) f_{2}$, où $\psi$ est une fonction de classe $C_{M}$ satisfaisant $\psi \equiv 1$ au voisinage de $E_{1}$ et $\psi \equiv 0$ au voisinage de $E_{2}$ (une telle fonction se construit facilement à l'aide des résultats de [B], §2). 
Dans le cas général, soit $g:=f_{1}-f_{2}$. Alors $g$ appartient à $C_{M}\left(\mathbb{R}^{n}\right)$ et est plate sur $E_{1} \cap E_{2}$. Par des arguments standard (voir par exemple [Dr], prop. 2.4) on a donc, pour tout multi-indice $L$,

$$
\left|D^{L} g(x)\right| \leq C^{l+1} l ! M_{l} h_{M}(C d),
$$

avec $d=d\left(x, E_{1} \cap E_{2}\right), x$ quelconque dans $\mathbb{R}^{n}, C$ désignant une constante positive convenable. Compte tenu de (1.1.6) et (2.1.3), il vient, quitte à augmenter $C$,

$$
\left|D^{L} g(x)\right| \leq C^{l+1} l ! N_{l}\left(h_{M}(B C d)\right)^{2} .
$$

On applique alors le lemme 2.3 avec $\lambda=B C$. Par la formule de Leibniz et (2.3.4), on vérifie alors que l'on a, toujours quitte à augmenter $C$,

$$
\left|D^{L}\left(\psi_{\lambda} g\right)(x)\right| \leq C^{l+1} l ! N_{l} h_{M}(\lambda d)
$$

pour tout $x$ de $\mathbb{R}^{n} \backslash\left(E_{1} \cap E_{2}\right)$ et tout multi-indice $L$.

Il s'ensuit que $\psi_{\lambda} g$ se prolonge en une fonction $\widetilde{g}$ de classe $C_{N}$ sur $\mathbb{R}^{n}$, plate sur $E_{1} \cap E_{2}$. On considère alors $f=\widetilde{g}+f_{2}$. Compte tenu de (2.1.3), on a bien $f \in C_{N}\left(\mathbb{R}^{n}\right)$; en outre, sur l'ouvert $\Omega_{j}(j=1,2)$ construit en (2.3), on a $f=f_{j}$ en vertu de (2.3.2) et (2.3.3). Il en résulte $\Re_{E_{j}} f=\Re_{E_{j}} f_{j}=F_{j}$.

$(\mathrm{B}) \Rightarrow(\mathrm{A})$. Supposons que $E_{1}$ et $E_{2}$ ne soient pas $\theta$-situés. Alors il existe une suite $\left(x^{j}\right)_{j \geq 0}$ de points de $\mathbb{R}^{n}$ telle que l'on ait $d\left(x^{j}, E_{1}\right)+d\left(x^{j}, E_{2}\right)<$ $\frac{1}{j} \theta\left(d\left(x^{j}, E_{1} \cap E_{2}\right)\right)$. Soit $\pi^{j}$ un point de $E_{1}$ tel que l'on ait $\left|\pi^{j}-x^{j}\right|=$ $d\left(x^{j}, E_{1}\right)$. A l'aide de (1.6.4) et d'inégalités élémentaires on établit facilement que pour $j$ assez grand, on a

$$
0<d\left(\pi^{j}, E_{2}\right) \leq \frac{C}{j} \theta\left(d\left(\pi^{j}, E_{1} \cap E_{2}\right)\right),
$$

où $C$ est une constante convenable. Modulo extraction, on peut alors supposer que $\left(\pi^{j}\right)_{j \geq 0}$ converge vers un point de $E_{1} \cap E_{2}$.

Soient les boules $B_{j}=B\left(\pi^{j}, \frac{1}{4} d\left(\pi^{j}, E_{1} \cap E_{2}\right)\right)$ et $B_{j}^{*}=B\left(\pi^{j}, \frac{1}{2} d\left(\pi^{j}\right.\right.$, $\left.\left.E_{1} \cap E_{2}\right)\right)$. Quitte à extraire encore une sous-suite, on peut supposer que les $B_{j}^{*}$ ne se coupent pas deux à deux. En utilisant de nouveau [B], §2, on montre qu'il existe $\varphi_{j} \in C_{M}\left(\mathbb{R}^{n}\right)$ telle que l'on ait

$$
0 \leq \varphi_{j} \leq 1, \quad \varphi_{j} \equiv 1 \quad \operatorname{sur} B_{j}, \quad \operatorname{supp} \varphi_{j} \subset B_{j}^{*}
$$

et

$$
\left|D^{L} \varphi_{j}(x)\right| \leq\left(C^{\prime}\right)^{l+1} l ! M_{l}\left(h_{M}\left(d\left(\pi^{j}, E_{1} \cap E_{2}\right)\right)\right)^{-1}
$$

pour tout multi-indice $L$ et tout $x$ de $\mathbb{R}^{n}$, où $C^{\prime}$ désigne une constante convenable. On pose alors, pour $\eta$ réel positif à déterminer,

$$
\phi(x)=\sum_{j=0}^{\infty} h_{N}\left(\theta\left(\eta d\left(\pi^{j}, E_{1} \cap E_{2}\right)\right)\right) \varphi_{j}(x) .
$$


Compte tenu de (2.1.2) et (1.1.6), il est facile de voir que $\phi$ définit une fonction de $C_{M}\left(\mathbb{R}^{n}\right)$, plate sur $E_{1} \cap E_{2}$, pourvu que le paramètre $\eta$ soit choisi assez petit.

Soit alors $F_{1}:=\Re_{E_{1}} \phi$. On a $F_{1} \in J_{M}\left(E_{1}\right)$. On pose $F_{2}=0$ de sorte que l'on a aussi $F_{2} \in J_{M}\left(E_{2}\right)$ et $\Re_{E_{1} \cap E_{2}} F_{1}=\Re_{E_{1} \cap E_{2}} F_{2}$.

Supposons maintenant que l'on puisse trouver une fonction $f$ de classe $C_{N}$ dans $\mathbb{R}^{n}$, satisfaisant $\Re_{E_{j}} f=F_{j}$ pour $j=1,2$. Comme $F_{2}$ est nul, $f$ doit être plate sur $E_{2}$, d'où l'on tire facilement

$$
|f(x)| \leq C^{\prime \prime} h_{N}\left(C^{\prime \prime} d\left(x, E_{2}\right)\right),
$$

pour tout $x$ de $\mathbb{R}^{n}$. Or on a $f\left(\pi^{j}\right)=\phi\left(\pi^{j}\right)=h_{N}\left(\theta\left(\eta d\left(\pi^{j}, E_{1} \cap E_{2}\right)\right)\right)$ par construction. Il en résulte, d'après (1.6.5) et (2.4.1), que pour une constante $T$ convenable, $T \geq 1$, on a

$$
\left|f\left(\pi^{j}\right)\right| \geq h_{N}\left(\eta^{\prime} j d\left(\pi^{j}, E_{2}\right)\right)
$$

avec $\eta^{\prime}=(C \bar{\theta}(T / \eta))^{-1}$. Ceci contredit clairement (2.4.2) et achève la preuve.

Remarque. L'inspection de ce qui précède montre que le rôle du lemme 2.3 consiste à produire des "multiplicateurs" pour les fonctions de classe $C_{M}$ plates sur $E_{1} \cap E_{2}$. Sur ces questions, on se reportera à $[\mathrm{M}] \mathrm{ou}$ [T] dans le cas $C^{\infty}$. Dans le cas des classes ultradifférentiables, 2.2 améliore, en un certain sens, un résultat de A. Lambert [L], V.1.2, qui permet de traiter uniquement le cas $M=N, \theta(t)=t$, sous des hypothèses techniques additionnelles.

2.5. Optimalité du théorème précédent. Dans le sens $(\mathrm{A}) \Rightarrow(\mathrm{B}) \mathrm{du}$ théorème 2.4 , la perte de régularité liée à la condition $(\mathcal{C})$ est en général la meilleure possible, comme on peut le voir en reprenant un exemple de $\mathrm{W}$. Pleśniak $[\mathrm{P}]$ :

Soit $m$ un réel, $m \geq 1$. On pose $E_{1}=\left\{(x, y) \in \mathbb{R}^{2}: 0 \leq x \leq 1, x^{m} \leq y\right.$ $\leq 1\}$ et $E_{2}=[0,1] \times[-1,0]$ dans $\mathbb{R}^{2}$. Les compacts $E_{1}$ et $E_{2}$ sont clairement $\theta$-situés, avec $\theta(t)=t^{m}$.

On considère la fonction $\varphi(x, y)=\exp (-1 / x)$ pour $(x, y)$ dans $E_{1}$. Elle est de classe $C_{M}$ avec $M_{l}=l^{l}$ (ou, indifféremment, $l$ !). En outre, comme $E_{1}$ a la propriété $(\mathrm{P})$ de Whitney (voir $(1.5)$ ), cette fonction définit un jet $F_{1}$ de $J_{M}\left(E_{1}\right)$. On prend pour $F_{2}$ le jet nul sur $E_{2}$. Alors $F_{1}$ et $F_{2}$ coïncident (au sens des jets) sur $E_{1} \cap E_{2}=\{(0,0)\}$.

$\mathrm{Au}$ vu de l'exemple 2.1(i), le théorème 2.4 stipule qu'il existe alors une fonction $f$ de $C_{M^{m}}\left(\mathbb{R}^{n}\right)$ satisfaisant $\Re_{E_{j}} f=F_{j}$ pour $j=1,2$ et, en particulier, $\left.f\right|_{E_{1}}=\varphi,\left.f\right|_{E_{2}}=0$.

Cependant, les arguments de $[\mathrm{P}], 1.9$, montrent que toute fonction $f$, de classe $C^{\infty}$ au voisinage de $E_{1} \cup E_{2}$ et supposée satisfaire ces deux dernières 
conditions, doit nécessairement vérifier, pour une constante $C$ convenable,

$$
\sup _{(x, y) \in \mathbb{R}^{2}}\left|\frac{\partial^{l} f}{\partial y^{l}}(x, y)\right| \geq C^{l} l ! l^{m l}
$$

pour tout entier $l$ : ceci montre que le résultat précédent est optimal.

On peut donner un éclairage général sur l'exemple 2.5 en considérant le corollaire suivant du théorème 2.4. Ce corollaire généralise, pour certains ouverts $\Omega$, la propriété d'extension de fonctions de la remarque 1.5.

2.6. Corollaire. Soient $\theta$ une fonction admissible et $M, N$ deux suites fortement régulières telles que la condition $(\mathcal{C})$ soit vérifiée. Soit $\Omega$ un ouvert borné de $\mathbb{R}^{n}$. On suppose que l'on a $\Omega=\Omega_{1} \cup \Omega_{2}$ où $\Omega_{1}$ et $\Omega_{2}$ sont deux ouverts dont l'adhérence a la propriété $(\mathrm{P})$ de Whitney. Alors les propriétés $\left(\mathrm{A}^{\prime}\right)$ et $\left(\mathrm{B}^{\prime}\right)$ suivantes sont équivalentes:

(A') Les compacts $\bar{\Omega}_{1}$ et $\bar{\Omega}_{2}$ sont $\theta$-situés.

$\left(\mathrm{B}^{\prime}\right)$ Toute fonction $f$ de $C_{M}(\bar{\Omega})$ s'étend en une fonction $\widetilde{f}$ de $C_{N}\left(\mathbb{R}^{n}\right)$ (c'est-à-dire que l'on a $\left.\widetilde{f}\right|_{\bar{\Omega}}=f$ ).

Preuve. $\left(\mathrm{A}^{\prime}\right) \Rightarrow\left(\mathrm{B}^{\prime}\right)$. Comme $\bar{\Omega}_{j}$ a la propriété $(\mathrm{P}),\left.f\right|_{\bar{\Omega}_{j}}$ définit un jet $F_{j}$ de $J_{M}\left(\bar{\Omega}_{j}\right)$ pour $j=1,2$ et on a clairement $\Re_{\bar{\Omega}_{1} \cap \bar{\Omega}_{2}} F_{1}=\Re_{\bar{\Omega}_{1} \cap \bar{\Omega}_{2}} F_{2}=$ $\Re_{\bar{\Omega}_{1} \cap \bar{\Omega}_{2}} f$. D'après 2.4 , il existe $\tilde{f}$ dans $C_{N}\left(\mathbb{R}^{n}\right)$ satisfaisant $\Re_{\bar{\Omega}_{j}} \widetilde{f}=F_{j}$ pour $j=1,2$ et donc $\left.\widetilde{f}\right|_{\bar{\Omega}}=f$.

$\left(\mathrm{B}^{\prime}\right) \Rightarrow\left(\mathrm{A}^{\prime}\right)$. Soient $F_{1}, F_{2}$ deux jets de $J_{M}\left(\bar{\Omega}_{1}\right)$ et $J_{M}\left(\bar{\Omega}_{2}\right)$ respectivement, satisfaisant $\Re_{\bar{\Omega}_{1} \cap \bar{\Omega}_{2}} F_{1}=\Re_{\bar{\Omega}_{1} \cap \bar{\Omega}_{2}} F_{2}$. D'après 1.4 , il existe deux fonctions $f_{j}$ de $C_{M}\left(\mathbb{R}^{n}\right)(j=1,2)$ telles que l'on ait $\Re_{\bar{\Omega}_{j}} f_{j}=F_{j}$. La condition de coïncidence sur $\bar{\Omega}_{1} \cap \bar{\Omega}_{2}$ montre que l'on peut définir une fonction $f$ de $C_{M}(\bar{\Omega})$ par recollement en posant $f=f_{j}$ sur $\bar{\Omega}_{j}$. Par hypothèse, il existe $\widetilde{f}$ dans $C_{N}\left(\mathbb{R}^{n}\right)$ telle que l'on ait $\left.\widetilde{f}\right|_{\bar{\Omega}}=f$, d'où l'on tire $\Re_{\bar{\Omega}_{j}} \widetilde{f}=F_{j}$ pour $j=1,2$. D'après 2.4 , ceci impose que $\bar{\Omega}_{1}$ et $\bar{\Omega}_{2}$ soient $\theta$-situés.

\section{Prolongement de fonctions}

3.1. DÉfinitions. Soit $\theta$ une fonction admissible. Un compact $E$ de $\mathbb{R}^{n}$ est dit $\theta$-régulier s'il existe une constante positive $\gamma$ telle que deux points quelconques $\xi$ et $x$ de $E$ puissent être joints par un arc rectifiable $\sigma$ contenu dans l'intérieur de $E$ sauf peut-être pour un nombre fini de points, et dont la longueur $|\sigma|$ vérifie

$$
\theta(|\sigma|) \leq \gamma|x-\xi|
$$

Par exemple, pour $\theta(t)=t^{m}, m \geq 1$, la $\theta$-régularité n'est autre que la $m$-régularité de Whitney $([\mathrm{M}],[\mathrm{T}],[\mathrm{P}])$. En particulier, pour $m=1$, on retrouve la propriété $(\mathrm{P})$, voir 1.5. Par ailleurs, de (1.6.7) résulte aussi le fait 
que si le compact $E$ est $\theta$-régulier, il est nécessairement $m$-régulier pour $m$ convenable. A ce titre, si $E$ est l'adhérence d'un ouvert borné $\Omega$, il s'ensuit que toute fonction de $C^{\infty}(\bar{\Omega})$ s'étend en une fonction de $C^{\infty}\left(\mathbb{R}^{n}\right)$ (voir $[\mathrm{M}]$, $[\mathrm{T}])$. On précisera en (3.4) le lien entre les notions de compacts $\theta$-situés et $\theta$-réguliers.

Soient alors $\theta$ une fonction admissible et $M, N$ deux suites fortement régulières. On dira que la condition $(\mathcal{D})$ est vérifiée s'il existe des constantes $b, c$, avec $0<b<1,0<c<1$, telles que l'on ait

$$
b h_{M}(l, c t) \leq \theta(t)^{l} N_{l}
$$

pour tout $l$ de $\mathbb{N}^{*}$ et tout $t$ de $\mathbb{R}_{+}$(voir (1.1.8) pour la définition de $h_{M}(l, \cdot)$ ).

Remarques. (i) Comme la condition $(\mathcal{C})$ (voir 2.1), la condition $(\mathcal{D})$ implique

$$
\sup _{l \geq 0}\left(M_{l} / N_{l}\right)^{1 / l}<\infty
$$

Il suffit pour le voir de prendre $t=1$ dans (3.1.2), en remarquant que l'on a $h_{M}(l, c)=c^{l} M_{l}$ pour $l$ assez grand.

(ii) En observant, plus précisément, que l'on a $h_{M}(l, t)=h_{M}(t)$ pour $t \leq M_{l} / M_{l+1}$ et $h_{M}(l, t)=t^{l} M_{l}$ pour $t>M_{l} / M_{l+1}$, on vérifie sans difficulté que si $t \rightarrow \theta(t) / t$ est croissante au voisinage de 0 et si la condition $(\mathcal{C})$ est satisfaite, alors la condition $(\mathcal{D})$ est également satisfaite.

(iii) Si $\theta$ satisfait les hypothèses (2.2.1) et (2.2.2) au voisinage de 0 dans $\mathbb{R}_{+}$et si $M$ est une suite fortement régulière donnée, il résulte de ce qui précède et de 2.2 qu'il existe toujours une suite $N$ fortement régulière telle que $(\mathcal{D})$ soit satisfaite.

Exemples. (i) Pour $\theta(t)=t^{m}, m \geq 1$, on vérifie aisément, à l'aide de (1.1.2), que $(\mathcal{D})$ est satisfaite avec $N=M^{m}$.

(ii) Le (ii) de la remarque précédente, joint à l'exemple 2.1(ii), montre que si $\theta(t)=t^{m}(\log (1+1 / t))^{-p}, m \geq 1, p \geq 0$, et si $M_{l}=l !^{\alpha}, \alpha>0$, alors $(\mathcal{D})$ est vérifiée avec $N_{l}=l !^{m \alpha}(\log l)^{l p}$.

3.2. ThÉORÈmE. Soient $\theta$ une fonction admissible et $M, N$ deux suites fortement régulières telles que la condition $(\mathcal{D})$ soit vérifiée. Soit $\Omega$ un ouvert borné de $\mathbb{R}^{n}$, d'adhérence $\theta$-régulière. Alors, pour toute fonction $f$ de $C_{M}(\bar{\Omega})$, il existe une fonction $\widetilde{f}$ de $C_{N}\left(\mathbb{R}^{n}\right)$ telle que l'on ait $\left.\widetilde{f}\right|_{\bar{\Omega}}=f$.

Preuve. On pose $F_{J}=D^{J} f$ pour tout multi-indice $J$. Vu le théorème 1.4, il s'agit de montrer que $F:=\left(F_{J}\right)_{J \in \mathbb{N}^{n}}$ est un jet de $J_{N}(\bar{\Omega})$. Compte tenu de (3.1.3), on a clairement l'estimation

$$
\left|F_{J}\right| \leq C^{j+1} j ! N_{j}
$$

pour une constante $C$ convenable. Il suffit donc de vérifier la condition de Taylor dans la définition des jets en 1.3. 
Soient $p$ un entier, $L$ un multi-indice avec $l \leq p$, $\xi$ et $x$ deux points de $\bar{\Omega}$. On considère $\sigma$ un arc rectifiable associé à $\xi$ et $x$ par la définition 3.1. Pour tout entier $q$ avec $q \geq p$, on a

$$
\left|F_{L}(x)-D^{L} T_{\xi}^{p} F(x)\right| \leq\left|E_{1}\right|+\left|E_{2}\right|
$$

avec

$$
E_{1}=F_{L}(x)-D^{L} T_{\xi}^{q} F(x) \quad \text { et } \quad E_{2}=D^{L}\left(T_{\xi}^{q} F-T_{\xi}^{p} F\right)(x) .
$$

A partir de [W], lemme 3, et par des arguments inspirés de [BBMT], 3.12, on obtient, quitte à augmenter $C$, l'estimation $\left|E_{1}\right| \leq C^{q+1} l ! M_{q+1}|\sigma|^{q+1-l}$. Avec l'inégalité triviale $|x-\xi| \leq|\sigma|$, on en déduit

$$
\left|E_{1}\right| \leq(C|\sigma|)^{q+1} M_{q+1} l !|x-\xi|^{-l} .
$$

Par ailleurs, on a

$$
\begin{aligned}
\left|E_{2}\right| & \leq \sum_{J, p+1 \leq j \leq q} \frac{1}{J !}\left|F_{J}(\xi) D^{L}(x-\xi)^{J}\right| \\
& \leq \sum_{\substack{J, p+1 \leq j \leq q \\
l_{1} \leq j_{1}, \ldots, l_{n} \leq j_{n}}} \frac{1}{(J-L) !} C^{j+1} j ! M_{j}|x-\xi|^{j-l} .
\end{aligned}
$$

A l'aide du fait élémentaire $j ! \leq 2^{j}(j-l) ! l ! \leq 2^{j} 2^{(n-1)(j-l)}(J-L) ! l !$, on en tire

$$
\left|E_{2}\right| \leq C\left(\sum_{j=p+1}^{q} 2^{-j}\left(2^{n+1} C|x-\xi|\right)^{j} M_{j}\right) l !\left(2^{n-1}|x-\xi|\right)^{-l} .
$$

Clairement, le maximum pour $p+1 \leq j \leq q+1$ de l'expression $\left(2^{n+1} C \mid x-\right.$ $\xi \mid)^{j} M_{j}$ est atteint pour $j=p+1$ ou pour $j=q+1$.

Dans le premier cas, on obtient

$$
\left|E_{2}\right| \leq C\left(\sum_{j=p+1}^{q} 2^{-j}\right)\left(2^{n+1} C|x-\xi|\right)^{p+1} M_{p+1} l !|x-\xi|^{-l},
$$

d'où, compte tenu de (3.1.3),

$$
\left|E_{2}\right| \leq C^{p+1} l ! N_{p+1}|x-\xi|^{p+1-l},
$$

quitte à augmenter $C$.

Dans le deuxième cas, on obtient similairement

$$
\left|E_{2}\right| \leq C\left(2^{n+1} C|x-\xi|\right)^{q+1} M_{q+1} l !|x-\xi|^{-l},
$$

d'où, en utilisant encore l'inégalité $|x-\xi| \leq|\sigma|$,

$$
\left|E_{2}\right| \leq C\left(2^{n+1} C|\sigma|\right)^{q+1} M_{q+1} l !|x-\xi|^{-l} .
$$


On choisit alors $q=q(p,|\sigma|)$ comme le plus petit entier supérieur ou égal à $p$ tel que l'on ait

$$
\left(2^{n+1} C|\sigma|\right)^{q+1} M_{q+1}=h_{M}\left(p+1,2^{n+1} C|\sigma|\right) .
$$

D'après (3.1.2), pour $|\sigma| \leq 1 / C^{\prime}$ avec $C^{\prime}=2^{n+1} c^{-1} C$, on a $h_{M}(p+1$, $\left.2^{n+1} C|\sigma|\right) \leq b^{-1} \theta\left(C^{\prime}|\sigma|\right)^{p+1} N_{p+1}$. Par (1.6.2) et (3.1.1), on a en outre $\theta\left(C^{\prime}|\sigma|\right) \leq \gamma \bar{\theta}\left(C^{\prime}\right)|x-\xi|$. On obtient donc des constantes $C^{\prime}, C^{\prime \prime}$ positives (dépendant de $f, \theta, \gamma, M, N)$ telles que l'on ait

$$
\left(2^{n+1} C|\sigma|\right)^{q+1} M_{q+1} \leq\left(C^{\prime \prime}\right)^{p+1} N_{p+1}|x-\xi|^{p+1}
$$

pour $|\sigma| \leq 1 / C^{\prime}$.

Finalement, (3.2.2) à (3.2.6) montrent que quels que soient les points $\xi$ et $x$ de $\bar{\Omega}$ satisfaisant $|\sigma| \leq 1 / C^{\prime}$, on a, quitte encore à augmenter $C$,

$$
\left|F_{L}(x)-D^{L} T_{\xi}^{p} F(x)\right| \leq C^{p+1} l ! N_{p+1}|x-\xi|^{p+1-l}
$$

pour tout entier $p$ et tout multi-indice $L$ avec $l \leq p$. Ceci termine la preuve (puisque pour $|\sigma|>1 / C^{\prime},|x-\xi|$ reste supérieur à la constante $\gamma^{-1} \theta\left(1 / C^{\prime}\right)$ et qu'alors l'inégalité de Taylor (3.2.7) est une simple conséquence de $(3.2 .1))$.

3.3. Remarques importantes. (i) La perte de régularité liée à la condition $(\mathcal{D})$ dans le théorème 3.2 n'est pas optimale en général, puisque $(\mathcal{D})$ reste vérifiée si l'on remplace $N$ par une suite $N^{\prime}$ telle que $\lim _{l \rightarrow \infty}\left(N_{l}^{\prime} / N_{l}\right)^{1 / l}=\infty$.

(ii) Il importe également de remarquer qu'étant donnés $\Omega$ un domaine borné de $\mathbb{R}^{2}, \theta$ une fonction admissible et $M, N$ deux suites fortement régulières telles que $(\mathcal{D})$ soit vérifiée, il n'est pas nécessaire que $\bar{\Omega}$ soit $\theta$ régulier pour avoir une propriété d'extension de $C_{M}(\bar{\Omega})$ à $C_{N}\left(\mathbb{R}^{n}\right)$. K. Wachta $[\mathrm{Wa}]$ a donné un exemple de domaine $\Omega_{0}$ de $\mathbb{R}^{2}$ tel que toute fonction de $C^{\infty}\left(\bar{\Omega}_{0}\right)$ s'étende en une fonction de $C^{\infty}\left(\mathbb{R}^{2}\right)$ bien que le compact $\bar{\Omega}_{0}$ ne soit pas Whitney-régulier, c'est-à-dire qu'il n'existe aucun $m(m \geq 1)$ tel que $\bar{\Omega}_{0}$ soit $\theta$-régulier pour $\theta(t)=t^{m}$. Reprenant cet exemple, on peut également montrer, à l'aide d'arguments similaires à la preuve de 3.2 , que toute fonction de $C_{M}\left(\bar{\Omega}_{0}\right)$ s'étend en une fonction de $C_{M^{4}}\left(\mathbb{R}^{2}\right),(\mathcal{D})$ étant vérifiée avec $N=M^{4}, \theta(t)=t^{4}$, et ceci bien que $\bar{\Omega}_{0}$ ne soit pas $\theta$-régulier.

La proposition suivante précise la relation entre compacts $\theta$-situés et $\theta$-réguliers.

3.4. Proposition. Soient $\theta$ une fonction admissible et $E_{1}, E_{2}$ deux compacts de $\mathbb{R}^{n}$. Alors :

(i) Si $E_{1} \cup E_{2}$ est $\theta$-régulier, les compacts $E_{1}$ et $E_{2}$ sont $\theta$-situés.

(ii) $S i E_{1}$ et $E_{2}$ sont $\theta$-situés avec $E_{1} \cap E_{2} \neq \emptyset$ et ont la propriété $(\mathrm{P})$ de Whitney (voir 1.5), le compact $E_{1} \cup E_{2}$ est $\theta$-régulier. 
Preuve. (i) Soient $\Omega$ un ouvert borné de $\mathbb{R}^{n}$ contenant $E_{1} \cup E_{2}, x$ un point de $\Omega$ et $x^{j}$ un point de $E_{j}$ tel que l'on ait $\left|x-x^{j}\right|=d\left(x, E_{j}\right)$, $j=1,2$. On a $\left|x^{1}-x^{2}\right| \leq d\left(x, E_{1}\right)+d\left(x, E_{2}\right)$. Or, par hypothèse, il existe un arc rectifiable $\sigma$ joignant $x^{1}$ à $x^{2}$ dans $E_{1} \cup E_{2}$ et tel que l'on ait $\theta(|\sigma|) \leq$ $\gamma\left|x^{1}-x^{2}\right|$, d'où

$$
\theta(|\sigma|) \leq \gamma\left(d\left(x, E_{1}\right)+d\left(x, E_{2}\right)\right) .
$$

Soit $\widehat{x}$ un point de $E_{1} \cap E_{2} \cap \sigma$. On note $\widehat{\sigma}$ le sous-arc de $\sigma$ joignant $x^{1}$ à $\widehat{x}$. On a clairement $d\left(x, E_{1} \cap E_{2}\right) \leq|x-\widehat{x}| \leq\left|x-x^{1}\right|+\left|x^{1}-\widehat{x}\right|$. En vertu des propriétés des fonctions admissibles on a alors

$$
\begin{aligned}
\theta\left(d\left(x, E_{1} \cap E_{2}\right)\right) & \leq \bar{\theta}(2) \bar{\theta}(\lambda)\left(\theta\left(\left|x-x^{1}\right| / \lambda\right)+\theta\left(\left|x^{1}-\widehat{x}\right| / \lambda\right)\right) \\
& \leq a \bar{\theta}(2) \lambda^{-1} \bar{\theta}(\lambda)\left|x-x^{1}\right|+\bar{\theta}(2) \bar{\theta}(\lambda) \theta\left(\left|x^{1}-\widehat{x}\right|\right),
\end{aligned}
$$

où l'on a posé $\lambda=\max \left(1, \sup _{x \in \Omega} d\left(x, E_{1}\right)+\operatorname{diam}\left(E_{1}\right)\right)$. Enfin, on a $\left|x^{1}-\widehat{x}\right| \leq$ $|\widehat{\sigma}| \leq|\sigma|$, d'où $\theta\left(d\left(x, E_{1} \cap E_{2}\right)\right) \leq a \bar{\theta}(2) \bar{\theta}(\lambda)\left(d\left(x, E_{1}\right)+\theta(|\sigma|)\right)$. On conclut alors, à l'aide de (3.4.1), que $E_{1}$ et $E_{2}$ sont $\theta$-situés.

(ii) Soient $\xi$ et $x$ des points de $E_{1} \cup E_{2}$. Il suffira de considérer le cas où l'on a $x \in E_{1}, \xi \in E_{2}$. On note $\widetilde{x}$ (resp. $\widetilde{\xi}$ ) un point de $E_{1} \cap E_{2}$ tel que l'on ait $|x-\widetilde{x}|=d\left(x, E_{1} \cap E_{2}\right)$ (resp. $\left.|\xi-\widetilde{\xi}|=d\left(\xi, E_{1} \cap E_{2}\right)\right)$. On sait qu'il existe dans $E_{1}$ (resp. $\left.E_{1}, E_{2}\right)$ un arc $\sigma_{1}$ joignant $x$ à $\widetilde{x}$ (resp. $\sigma_{2}$ joignant $\widetilde{x}$ à $\widetilde{\xi}$ et $\sigma_{3}$ joignant $\widetilde{\xi}$ à $\xi$ ), tel que l'on ait $\left|\sigma_{1}\right| \leq C|x-\widetilde{x}|$ (resp. $\left|\sigma_{2}\right| \leq C|\widetilde{x}-\widetilde{\xi}|$ et $\left.\left|\sigma_{3}\right| \leq C|\widetilde{\xi}-\xi|\right)$ pour une constante $C$ convenable. On a aussi $|\widetilde{x}-\widetilde{\xi}| \leq|\widetilde{x}-x|+|x-\xi|+|\xi-\widetilde{\xi}|$.

Soit $\sigma:=\sigma_{1} \cup \sigma_{2} \cup \sigma_{3}$. Des inégalités précédentes résulte, quitte à augmenter $C$, la majoration $|\sigma| \leq C(|x-\widetilde{x}|+|\xi-\widetilde{\xi}|+|x-\xi|)$ et par conséquent, en vertu des propriétés des fonctions admissibles, $\theta(|\sigma|) \leq C^{\prime}(\theta(|x-\widetilde{x}|)$ $+\theta(|\xi-\widetilde{\xi}|)+\theta(|x-\xi|))$ pour une constante $C^{\prime}$ convenable, ne dépendant que de la géométrie de $E_{1} \cup E_{2}$.

Or on a $\theta(|x-\widetilde{x}|)=\theta\left(d\left(x, E_{1} \cap E_{2}\right)\right) \leq \gamma^{-1} d\left(x, E_{2}\right) \leq \gamma^{-1}|x-\xi|$ (resp. $\left.\theta(|\xi-\widetilde{\xi}|) \leq \gamma^{-1}|x-\xi|\right)$ puisque $E_{1}$ et $E_{2}$ sont $\theta$-situés. Enfin on a $\theta(|x-\xi|) \leq C^{\prime \prime}|x-\xi|$ d'après (1.6.6). Il s'ensuit que l'arc rectifiable $\sigma$ joint $\xi$ à $x$ dans $E_{1} \cup E_{2}$ et satisfait $\theta(|\sigma|) \leq C^{\prime}|x-\xi|$ quitte à augmenter $C^{\prime}$. Enfin $\sigma$ est contenu dans l'intérieur de $E_{1} \cup E_{2}$ sauf éventuellement pour un nombre fini de points puisque c'est le cas pour $\sigma_{1}, \sigma_{2}, \sigma_{3}$. Ceci achève le preuve.

3.5. Un lien avec les résultats $d u \S 2$. Si $\Omega$ est un ouvert satisfaisant les hypothèses du sens $\left(\mathrm{A}^{\prime}\right) \Rightarrow\left(\mathrm{B}^{\prime}\right)$ de 2.6 , il résulte de 3.4 (ii) que $\bar{\Omega}$ est $\theta$-régulier, donc, d'après 3.2 , que la propriété d'extension de $C_{M}(\bar{\Omega})$ à $C_{N}\left(\mathbb{R}^{n}\right)$ décrite dans le sens direct de 2.6 reste valable si l'on remplace la condition $(\mathcal{C})$ par $(\mathcal{D})$. Ceci peut aussi être vu comme conséquence du résultat général 
suivant, que l'on peut établir par des arguments identiques à ceux de la preuve de 3.2 :

On suppose que la condition $(\mathcal{D})$ est satisfaite. Soient $E_{1}, \ldots, E_{k}$ des compacts de $\mathbb{R}^{n}$ et, pour $1 \leq j \leq k$, des jets $F_{j}$ de $J_{M}\left(E_{j}\right)$ tels que l'on ait $\Re_{E_{i} \cap E_{j}} F_{i}=\Re_{E_{i} \cap E_{j}} F_{j}$ pour $1 \leq i \leq k, 1 \leq j \leq k, i \neq j$. Alors, si les $E_{j}$ sont $\theta$-situés deux à deux, il existe une fonction $f$ de $C_{N}\left(\mathbb{R}^{n}\right)$ telle que l'on ait $\Re_{E_{j}} f=F_{j}$ pour $1 \leq j \leq k$.

Lorsque $t \rightarrow \theta(t) / t$ est croissante au voisinage de 0 dans $\mathbb{R}_{+}$, ce résultat contient, en vertu de la remarque 3.1(ii), le sens $(\mathrm{A}) \Rightarrow(\mathrm{B})$ du théorème 2.4 et, en conséquence, le sens $\left(\mathrm{A}^{\prime}\right) \Rightarrow\left(\mathrm{B}^{\prime}\right)$ du corollaire 2.6. Mais il n'est plus question de réciproque ici.

Il est à noter enfin que la preuve de 3.2 et du résultat précédent n'utilisent pas la forte régularité de $M$ (mais seulement sa convexité logarithmique (1.1.1)). La forte régularité de $N$ n'est, quant à elle, utilisée que dans l'étape finale qui consiste, via le théorème 1.4 , à étendre le jet de classe $J_{N}$ obtenu.

3.6. A propos de la notion de fonction admissible. On commente pour conclure les hypothèses en 1.6.

Les conditions (1.6.1) et (1.6.3) garantissent la non-vacuité des notions d'ensembles $\theta$-situés ou $\theta$-réguliers.

La condition (1.6.2) signifie que la décroissance de $\theta(t)$ quand $t$ tend vers 0 n'est pas trop rapide, voir (1.6.7). Si on supprime (1.6.2), toute propriété d'extension tombe généralement en défaut, comme le montre l'exemple suivant :

On pose $\theta(t)=\exp (-1 / t), \Omega_{1}=\left\{(x, y) \in \mathbb{R}^{2}: 0<x<1, \exp (-1 / x)<\right.$ $y<1\}$ et $\left.\Omega_{2}=\right] 0,1[\times]-1,0\left[\right.$ dans $\mathbb{R}^{2}$. Alors $\bar{\Omega}_{1}$ et $\bar{\Omega}_{2}$ sont $\theta$-situés. En outre, si on pose $\varphi(x, y)=\exp (-1 / x)$ pour $(x, y) \in \Omega_{1}$ et $\varphi(x, y)=0$ pour $(x, y) \in \Omega_{2}$, on définit ainsi une fonction de $C_{M}(\bar{\Omega})$ pour $M_{l}=l^{l}$ et $\Omega=\Omega_{1} \cup \Omega_{2}$. Cependant il est classique ([Bi], 2.18) que $\varphi$ ne peut même pas s'étendre en une fonction de classe $C^{\infty}$ sur $\mathbb{R}^{2}$.

\section{Références}

[Bi] E. Bierstone, Differentiable functions, Bol. Soc. Brasil. Mat. 11 (1980), 139-190.

[BBMT] J. Bonet, R. W. Braun, R. Meise and B. A. Taylor, Whitney's extension theorem for nonquasianalytic functions, Studia Math. 99 (1991), 156-184.

[B] J. Bruna, An extension theorem of Whitney type for non-quasianalytic classes of functions, J. London Math. Soc. 22 (1980), 495-505.

[CC] J. Chaumat et A.-M. Chollet, Théorème de Whitney dans des classes ultradifférentiables, Publ. Inst. Rech. Math. Lille 28 (1992), VIII.1-VIII.31 et C. R. Acad. Sci. Paris 315 (1992), 901-906. 
[Dr] B. Droste, Holomorphic approximation of ultradifferentiable functions, Math. Ann. 257 (1981), 293-316.

[L] A. Lambert, Quelques théorèmes de décomposition des ultradistributions, Ann. Inst. Fourier (Grenoble) 29 (3) (1979), 57-100.

[M] B. Malgrange, Ideals of Differentiable Functions, Tata Institute for Fundamental Research, Bombay and Oxford Univ. Press, 1966.

[P] W. Pleśniak, Extension and polynomial approximation of ultradifferentiable functions in $\mathbb{R}^{N}$, Bull. Soc. Roy. Sci. Liège 63 (1994), 393-402.

[T] J.-C. Tougeron, Idéaux de fonctions différentiables, Ergeb. Math. Grenzgeb. 71, Springer, 1972.

[Wa] K. Wachta, Prolongation des fonctions $C^{\infty}$, Bull. Polish Acad. Sci. Math. 31 (1983), 245-248.

[W] H. Whitney, Functions differentiable on the boundaries of regions, Ann. of Math. 35 (1934), 482-485.

CNRS-URA 751

MATHÉMATIQUES-BÂT. M2

UNIVERSITÉ DES SCIENCES ET TECHNOLOGIES DE LILLE

F-59655 VILLENEUVE D'ASCQ CEDEX, FRANCE

E-mail: THILLIEZ@GAT.UNIV-LILLE1.FR

Reçu par la Rédaction le 19.12.1994 